\title{
HOLOMORPHIC MAPPINGS OF DOMAINS WITH GENERIC CORNERS
}

\author{
S. M. WEBSTER
}

\begin{abstract}
The boundary behavior of a biholomorphic mapping $f$ between two domains with real analytic, generic, nondegenerate corners in $\mathbf{C}^{n}$ is considered. Under certain minimal regularity assumptions on $f$ it is shown that $f$ continues holomorphically past the boundary.
\end{abstract}

Introduction. The problem of extending a holomorphic mapping between two domains with smooth boundaries in the complex space $\mathbf{C}^{n}$ has received considerable attention in recent years. In this note we consider the continuation problem for a mapping $f$ defined on a domain which has corners of a certain kind. We shall show that $f$ can be analytically extended by means of a reflection principle, provided it satisfies certain minimal initial regularity conditions. The main point here is that an argument due to $\mathrm{H}$. Lewy, when suitably modified, gives holomorphic continuation in a much more general situation.

Let $D$ be a domain in $\mathbf{C}^{n}$ and $U$ an open set which meets the boundary of $D$. Let $r^{i}(z), 1 \leqslant i \leqslant l$, where $1 \leqslant l \leqslant n$, be twice continuously differentiable real valued functions defined on $U$ for which $d r^{1} \wedge \cdots \wedge d r^{l} \neq 0$ and

$$
D \cap U=\left\{z \in U: r^{i}(z)<0,1 \leqslant i \leqslant l\right\} \text {. }
$$

The manifold

$$
M=\left\{z \in U: r^{i}(z)=0,1 \leqslant i \leqslant l\right\}
$$

is a generic corner of $D$ if also $\partial r^{1} \wedge \cdots \wedge \partial r^{l} \neq 0$ on $M$; i.e. the complex gradients of the $r^{i}$ should be independent. $M$ is a real submanifold of codimension $l$. The holomorphic tangent space $H_{z}(M), z \in M$, is the vector space of all vectors of type $(1,0)$ annihilating the defining functions $r^{i}$ at $z$. The condition means that $H_{z}$ has complex codimension $l$. Any real submanifold $M$ of $\mathbf{C}^{n}$ of codimension $l$ satisfying this condition is called a generic real submanifold. If $X$ and $Y$ are local sections of $H(M)$ near $z$, the Levi form of $M$ is defined by $(X, Y) \rightarrow L_{z}(X, Y) \equiv i[X, \bar{Y}]$, $\bmod H_{z} \oplus \bar{H}_{z}$. It is an hermitian bilinear form on $H_{z}$ with values in $T_{z} \otimes C / H_{z} \oplus \bar{H}_{z}$, where $T_{z}$ denotes the real tangent space of $M$ at $z . M$ is nondegenerate at $z$ if the linear mapping $Y \rightarrow L_{z}(\cdot, Y)$ is injective on $H_{z}$.

We may now state the main result.

Received by the editors February 18, 1981.

1980 Mathematics Subject Classification. Primary 32H99.

Key words and phrases. Biholomorphic map, reflection principle, generic submanifold, nondegenerate Levi form.

${ }^{1}$ Alfred P. Sloan Fellow. 
THEOREM. Let $D$ be a domain in $\mathbf{C}^{n}$ with a generic real analytic corner $M$, and let $M^{\prime}$ be a generic analytic real submanifold with nondegenerate Levi form in $\mathbf{C}^{m}$. Suppose $f$ is a holomorphic mapping from $D$ to $\mathbf{C}^{m}$, which together with its first derivatives extends continuously to $M$, taking $M$ into $M^{\prime}$. If at some point $z$ of $M$ the differential of $f$ induces a linear isomorphism of the holomorphic tangent spaces $H_{z}(M)$ and $H_{f(z)}\left(M^{\prime}\right)$, then $f$ continues holomorphically to a full neighborhood of $z$ in $\mathbf{C}^{n}$.

REMARKS. (a) It is not required that the hypersurfaces $r^{i}=0$ be analytic. It will be clear from the proof that the requirement that $D$ have a corner along $M$ is too strong. However, the points of $M$ must be accessible via suitable cones lying in $D$. In one complex variable there are no generic corners other than smooth curves.

(b) The hypotheses imply that $M$ and $M^{\prime}$ have the same holomorphic dimension. In case $M$ is totally real it is not necessary to assume that the first derivatives of $f$ extend continuously to $M$. The result then follows from the edge-of-the-wedge theorem after a change of coordinates.

(c) The theorem and its proof given below reduce to those given by $\mathrm{H}$. Lewy in [1], when $n=m$ and $M$ and $M^{\prime}$ are both hypersurfaces. If, in addition, $M$ and $M^{\prime}$ are both strongly pseudoconvex and $f$ is biholomorphic, then the theorem, which is purely local, was proved in [2] under the assumption that $f$ is Hölder continuous with exponent $\frac{1}{2}+\varepsilon, \varepsilon>0$. See also [5] and Pinchuk [3].

1. Reflection about a generic, nondegenerate submanifold. Let $M \subset \mathrm{C}^{n}$ be an analytic generic real submanifold of codimension $l, 1 \leqslant l \leqslant n$. Choose a neighborhood $U$ and local real analytic defining functions $r^{i}=r^{i}(z, \bar{z})$ for $M$ as in (0.2). We may assume that the power series $r^{i}(z, \bar{w})$ converge for $z, w \in U$. Following [5] we define local nonsingular complex varieties $Q_{z}$ of codimension $l$ by $Q_{z}=\{w \in$ $\left.U: r^{i}(z, \bar{w})=0,1 \leqslant i \leqslant l\right\}$. Because of the reality condition on the $r^{i}, w \in Q_{z} \Leftrightarrow z$ $\in Q_{w}$. Given $z$ near $z_{0} \in M$ and a complex $(n-l)$-plane $p$ nearly parallel to $H_{z_{0}}(M)$, we try to determine a "reflected" point $w \in Q_{z}$ by requiring $T_{z} Q_{w}=p$. If we set $q=T_{w} Q_{z}$, the problem is to set up an antiholomorphic involution $(z, p) \leftrightarrow$ $(w, q)$ of pointed ( $n-l)$-planes. $p$ and $q$ are elements of the complex Grassmanian $\operatorname{Gr}(n-l, n)$ of $(n-l)$-planes in $\mathbf{C}^{n}$. Gr has complex dimension $l\left(n_{\bullet}-l\right)$, whereas the image of $w \rightarrow T_{z} Q_{w}$ has dimension at most $n-l$. Thus except for the hypersurface case $l=1, p$ and $q$ must satisfy some consistency condition.

We consider also the complexification of $M, M^{c}=\left\{(z, w) \in U \times U: r^{i}(z, \bar{w})=0\right.$, $1 \leqslant i \leqslant l\}$. With $z$ and $\eta=\bar{w}$ as variables, it is clear that $M^{c}$ is a complex submanifold of $\mathbf{C}^{2 n}$ of codimension $l$. There is a natural mapping $\pi$ from $M^{c}$ to $\mathrm{C}^{n} \times \mathrm{Gr}$ given by $\pi(z, w)=T_{z} Q_{w} . \pi$ is holomorphic in $z$ and antiholomorphic in $w$.

LEMMA. The mapping $\pi$ is an immersion at $\left(z_{0}, z_{0}\right), z_{0} \in M$, if and only if the Levi form of $M$ is nondegenerate at $z_{0}$.

Proof. This a matter of checking the definitions. We denote $\partial_{a}=\partial / \partial z^{a}$ and $\partial_{\bar{a}}=\partial / \partial \bar{w}^{a}, 1 \leqslant a \leqslant n$. By a linear change of coordinates we may assume

$$
\begin{aligned}
\operatorname{det}\left(\partial_{j} r^{i}\right) & \neq 0, \quad 1 \leqslant i, j \leqslant l \\
\partial_{\alpha} r^{i} & =0, \quad 1 \leqslant i \leqslant l, l<\alpha \leqslant n, \text { at }(z, \bar{w})=\left(z_{0}, \bar{z}_{0}\right) .
\end{aligned}
$$


We define the operators

$$
X_{\alpha}=\operatorname{det}\left[\begin{array}{c|c}
\partial_{\alpha} & \left(\partial_{j}\right) \\
\hdashline\left(\partial_{\alpha} r^{i}\right) & -\frac{-}{\left(\partial_{j} r^{i}\right)}
\end{array}\right](z, \bar{w})
$$

for each $\alpha, l<\alpha \leqslant n$, in which the $(l+1) \times(l+1)$ matrix is to be expanded across the top row. Clearly, the $X_{\alpha}$ are independent and annihilate the function $r^{i}(\cdot, \bar{w})$. They form a basis for the vectors of type $(1,0)$ tangent to $Q_{w}$ at $z$. A basis $X_{\bar{\alpha}}$ for the $(0,1)$-tangent space of $Q_{w}$ at $z$ is given by (1.2) with $\partial_{\alpha}$ and $\partial_{j}$ replaced by $\partial_{\bar{\alpha}}$ and $\partial_{j}$, respectively. When $z=w \in M$, the $X_{\alpha}$ form a basis for $H_{z}(M)$. By (1.1) we may solve the equations $r^{i}(z, \bar{w})=0$ for $z^{i}, 1 \leqslant i \leqslant l$, in terms of $z^{\alpha}, l<\alpha \leqslant n$ :

$$
z^{i}=z^{i}\left(z^{\alpha}, \bar{w}\right), \quad p_{\alpha}^{i}=\frac{\partial z^{i}}{\partial z^{\alpha}}\left(z^{\alpha}, \bar{w}\right), \quad \partial_{\alpha} r^{i}+\sum_{k} p_{\alpha}^{k} \partial_{k} r^{i}=0 .
$$

The $p_{\alpha}^{i}$ are coordinates for the plane $p=T_{z} Q_{w}$. It is clear that $\pi:(z, w) \rightarrow(z, p)$ is an immersion at $\left(z_{0}, z_{0}\right)$ if and only if the $(n-l) \times l(n-l)$ matrix of derivatives (indexed by $(n-l) \beta$ 's and $l(n-l) \alpha j$ 's)

$$
\left(X_{\beta}^{-} p_{\alpha}^{j}\right)
$$

has rank $n-l$ when $z=w=z_{0}$. We want to show that this condition is equivalent to $M$ having a nondegenerate Levi form at $z_{0}$. By Cartan's formula for exterior derivative the Levi form has the coordinate representation

$$
\begin{aligned}
L(X, Y) & =\left(i \partial r^{1}([X, \bar{Y}]), \ldots, i \partial r^{l}([X, \bar{Y}])\right) \\
& =-i\left(\partial \bar{\partial} r^{1}(X, \bar{Y}), \ldots, \partial \bar{\partial} r^{l}(X, \bar{Y})\right) .
\end{aligned}
$$

We write

$$
\partial \bar{\partial} r^{j}(X, \bar{Y})=\sum \xi^{a} \bar{Y}\left[\partial_{a} r^{j}\right], \quad X=\sum \xi^{a} \partial_{a} .
$$

Since $\bar{Y}$ is a linear combination of the $X_{\bar{\alpha}}$, and $X_{\alpha}=\partial_{\alpha}$ at $\left(z_{0}, \bar{z}_{0}\right)$, the nondegeneracy of the Levi form is equivalent to the matrix $\left(X_{\beta}^{-}\left[\partial_{\alpha} r^{j}\right]\right)$ having rank $n-l$. If we differentiate the last equation in (1.3) with $X_{\beta}^{-}$and use the fact that $p_{\alpha}^{k}\left(z_{0}, \bar{z}_{0}\right)=0$ and (1.1), we see that this matrix has the same rank as (1.4).

Let $\tilde{M}^{c}$ denote the image of $\pi$. Since $\pi$ is holomorphic in $(z, \eta=\bar{w})$ and an immersion, $\tilde{M}^{c}$ is a (local) complex submanifold of $\mathbf{C}^{n} \times \mathrm{Gr}$ of dimension $2 n-l$. By the reality condition on the $r^{i}, M^{c}$ is invariant under the antiholomorphic involution $(z, \bar{w}) \rightarrow(w, \bar{z})$. This reflection induces a reflection on $\tilde{M}^{c}$ via $\pi$ as follows. Given $(w, q) \in \tilde{M}^{c},(w, q)=\pi(w, z)$ for a (locally) unique $z$. Use equation (1.3) with argument $(z, \bar{w})$ to define $p$. It is clear that the correspondence $(z, p) \rightarrow$ $(w, q)$ is antiholomorphic and involutive.

2. Application to holomorphic mappings. In this section we prove the theorem. Let $D \cap U$ and $M$ be given by (0.1) and (0.2), respectively. We first make a local coordinate change in a neighborhood of the particular point $z \in M$. After a translation and rotation we may assume that this $z=0$ and that $T_{0}(M)$ is given by $y^{j} \equiv \operatorname{Im} z^{j}=0,1 \leqslant j \leqslant l$, and that $H_{0}(M)$ is given by $z^{j}=0,1 \leqslant j \leqslant l$. So $z^{\alpha}$, $l<\alpha \leqslant n$, are coordinates on $H_{0}(M)$, and $z^{\alpha}, x^{i} \equiv \operatorname{Re} z^{j}$, are coordinates on $T_{0}(M)$. 
Locally, as a graph over $T_{0}(M), M$ is given by equations

$$
\rho^{j}(z, \bar{z}) \equiv-y^{j}+h^{j}\left(z^{\alpha}, \bar{z}^{\alpha}, x^{i}\right)=0, \quad 1 \leqslant j \leqslant l,
$$

in which the $h^{j}$ are convergent power series about the origin which vanish together with their first derivatives when $z^{\alpha}=x^{i}=0$. We define a real analytic local coordinate change $T:\left(\zeta^{j}, \zeta^{\alpha}\right) \rightarrow\left(z^{j}, z^{\alpha}\right)$, which is holomorphic in the $\zeta^{j}$ when the $\zeta^{\alpha}$ are held constant, by

$$
T: \begin{aligned}
& z^{\alpha}=\zeta^{\alpha} \\
& z^{j}=\zeta^{j}+i h^{j}\left(\zeta^{\alpha}, \bar{\zeta}^{\alpha}, \zeta^{j}\right) .
\end{aligned}
$$

It is clear that the (real) Jacobian determinant does not vanish at the origin, and that $\operatorname{Im} \zeta^{i}=0$ corresponds to $M$. For the functions $r^{i}$ defining $D$ the sets of covectors $\left\{\partial r^{i}\right\}$ and $\left\{\partial \rho^{i}\right\}$ in the $z$-coordinate system have the same linear span at points of $M$. Since $\bar{\partial}_{\zeta} z^{j}=0$ at the origin of the $\zeta$-system, $\left\{\partial_{\zeta} r^{j}\right\}$ and $\left\{d \zeta^{j}\right\}$ have the same span there. Since the first order approximatioin of $D$ in the $\zeta$ system is the linear corner $\left\{d_{\zeta} r^{j}<0\right\}$, it is clear that by a complex linear change of the $\zeta^{j}, 1 \leqslant j \leqslant l, D$ can be made to contain the wedge $W^{+}=\left(U_{1}+i V^{+}\right) \times U_{0}$. Here $U_{0}$ is a neighborhood of $\zeta^{\alpha}=0$ in the $\zeta^{\alpha}$-space, $U_{1}$ is a neighborhood of $\operatorname{Re} \zeta^{j}=0$ in the $\operatorname{Re} \zeta^{j}$-space, and $V^{+}$ is the (truncated) cone $\operatorname{Im} \zeta^{j}>0,1 \leqslant j \leqslant l$, in the $\operatorname{Im} \zeta^{j}$-space. We denote by $V^{-}$the (symmetrically truncated) cone $\operatorname{Im} \zeta^{j}<0,1 \leqslant j \leqslant l$, and by $W^{-}$the corresponding wedge. If $z=\left(z^{j}, c^{\alpha}\right)=T\left(\zeta^{j}, c^{\alpha}\right)$ and $w=\left(w^{j}, c^{\alpha}\right)=T\left(\bar{\zeta}^{j}, c^{\alpha}\right)$, it is clear from (2.1) and (2.2) that $\rho^{j}(\mathrm{z}, \bar{w})=0$, and that these equations characterize the reflection $\zeta^{j} \rightarrow \bar{\zeta}^{j}$.

Now we use the above to extend the mapping $f$. Let $\eta=\left(\eta^{j}, c^{\alpha}\right)$ be a point of $W^{-}, \zeta=\left(\bar{\eta}^{j}, c^{\alpha}\right), w=T(\eta), z=T(\zeta)$. Then $z \in D$ and $\rho^{j}(z, \bar{w})=0$; i.e. $z \in Q_{w}$. Let $p=T_{z} Q_{w}, z^{\prime}=f(z)$, and $p^{\prime}=d f_{z}(p)$. As $\operatorname{Im} \eta^{j} \rightarrow 0$, it follows that $z \rightarrow z_{0} \in M$, and $p \rightarrow H_{z_{0}}(M)$, for some $z_{0}$. Since $f$ is $C^{1}$ and $d f$ is an isomorphism on $H(M)$, it follows that $p^{\prime}$ is an $(n-l)$-plane which approaches $p_{0}^{\prime}=H_{z_{0}^{\prime}}\left(M^{\prime}\right), z_{0}^{\prime}=f\left(z_{0}\right)$. In order to reflect $\left(z^{\prime}, p^{\prime}\right)$ by the method of $\S 1$, we must show that $\left(z^{\prime}, p^{\prime}\right) \in \tilde{M}^{\prime c}$. Let $\Psi^{\prime}\left(z^{\prime}, p^{\prime}\right)=0$ denote (local) holomorphic functions defining the complex manifold $\tilde{M}^{\prime c}$. Since $\left(z_{0}^{\prime}, p_{0}^{\prime}\right) \in \tilde{M}^{\prime c}$, we see that $\Psi^{\prime}\left(z^{\prime}, p^{\prime}\right) \rightarrow 0$ as $\operatorname{Im} \eta^{j} \rightarrow 0$. By construction $\Psi^{\prime}\left(z^{\prime}, p^{\prime}\right)$ is an antiholomorphic function of $\eta^{j}$, for $\eta^{\alpha}=c^{\alpha}$ fixed, on $W^{-}$. We extend this function continuously to $W \equiv W^{+} \cup W^{-}$by setting it equal to 0 on $W^{+}$. By the edge-of-the-wedge theorem (see [4]) $\Psi^{\prime}$ continues holomorphically to a full neighborhood of $\left(0, c^{\alpha}\right)$ in the $\zeta^{\alpha}$-space. Since it vanishes on the real axis, it is identically zero. Hence, for $\operatorname{Im} \eta^{j}$ sufficiently small, uniformly in $c^{\alpha},\left(z^{\prime}, p^{\prime}\right) \in \tilde{M}^{\prime c}$, and the reflected point-plane $\left(w^{\prime}, p^{\prime}\right)$ is defined and holomorphic in $\eta^{j} \in W^{-}$, for each fixed $c^{\alpha}$.

Thus $\eta \rightarrow w^{\prime}\left(\eta^{j}, c^{\alpha}\right)$ gives a continuous extension $\tilde{f}$ of $f \circ T$ to $W$. We now get an extension $\tilde{F}$ of $\tilde{f}$ to a full neighborhood of 0 in $C^{n}$. This is given by the following integral, formula (6), $\$ 4$, of [4],

$$
2 \pi \tilde{F}\left(\zeta^{j}, c^{\alpha}\right)=\int_{-\pi}^{\pi} \tilde{f}\left(\Phi\left(\zeta^{i}, e^{i \theta}\right), c^{\alpha}\right) d \theta .
$$

$\tilde{F}$ is continuous in $\left(\zeta^{j}, c^{\alpha}\right)$ since $\tilde{f}$ is continuous on $W$. It is also holomorphic on each plane $\zeta^{\alpha}=c^{\alpha}$. Set $F=\tilde{F} \circ T^{-1}$. $F$ continuously extends $f$ to a neighborhood of the original point of $M$ and is holomorphic on each $l$-plane $\zeta^{\alpha}=c^{\alpha}$. As in [1] (or see [2]), 
we can argue that $F$ is holomorphic as follows. Let $I_{c}(F)$ be the complex line integral of $F$ about a small loop $c$ in the complex $z^{\alpha}$-plane. $I_{c}(F)$ is holomorphic in $z^{j}$ and vanishes for the open set of $z^{j}$ for which $\left(z^{j}, c\right) \subset D$. Hence, $I_{c}(F) \equiv 0$, and $F$ is holomorphic in $z^{\alpha}$ by Morera's theorem.

\section{REFERENCES}

1. H. Lewy, On the boundary behavior of holomorphic mappings, Contrib. Centro Linceo Inter. Sc. Mat. e Loro Appl. No. 35, Acad. Naz. dei Lincei, 1977, pp. 1-8.

2. L. Nirenberg, S. Webster and P. Yang, Local boundary regularity of holomorphic mappings, Comm. Pure Appl. Math. 33 (1980), 305-338.

3. S. I. Pinchuk, On the analytic continuation of biholomorphic mappings, Math. Sb. 27 (3) (1975), 375-392.

4. W. Rudin, Lectures on the edge-of-the-wedge theorem, CBMS Regional Conf. Ser. in Math., no. 6, Amer. Math. Soc., Providence, R. I., 1971.

5. S. Webster, On the reflection principle in several complex variables, Proc. Amer. Math. Soc., 72 (1978), 26-28.

School of Mathematics, University of Minnesota, Minneapolis, Minnesota 55455 